\title{
Los dilemas de la representación política contemporánea en Bolivia: movimientos sociales, partido y Estado en tiempos de 'Proceso de Cambio'
}

\author{
The dilemmas of political representation in contemporary Bolivia: social movements, \\ party and State under the 'Process of Change'
}

\author{
Clayton Mendonça Cunha Filho \\ Post-doctorante del Instituto de Relaciones \\ Internacionales de la Universidad de Brasília (IREL/UnB), \\ Brasília, DF, Brasil \\ clayton.filho@gmail.com
}

\begin{abstract}
Resumen: Viviendo nueva estabilidad política bajo la hegemonía de un partido (MAS) que constituye su identidad en gran medida por la negación de su propio carácter partidario y declarándose mero instrumento político de los movimientos sociales, Bolivia se ve delante de importantes desafíos a la institucionalización de su política. Por un lado, es innegable que muchos de los más importantes movimientos sociales y organizaciones de la sociedad civil encuentran representación e influencia sobre la máquina estatal a través de dicho partido. Por otro, vuelven a la escena clásicas cuestiones de cooptación y autonomía de los movimientos sociales. Al mismo tiempo, la oposición se mantiene fluida e incapaz de organizar partidos estables y coherentes, reduciéndose muchas veces a algunas trincheras regionales localizadas. El objetivo de este trabajo es trazar un mapa del escenario político boliviano a partir del análisis de los principales temas y clivajes existentes en la agenda política contemporánea tras más de nueve años de gobierno del MAS y como los mismos se relacionan al partido gubernista, a la oposición y a los movimientos sociales de alcance nacional más importantes del país, bien como consideraciones sobre la primacía que tales movimientos ejercen en la intermediación política y su relación con los partidos tanto oficialista cuanto opositores.
\end{abstract}

Palabras clave: Bolivia; Representación política; Movimientos sociales; Proceso de cambio; Movimiento al socialismo.

Abstract:Living a new age of political stability under the hegemony of a party (MAS) that builds its identity to a great extent by denying its classification as a political party and declaring itself to be a mere instrument for the social movements, Bolivia faces important challenges to its political institutionalization. On the one hand, it is undeniable that most of the country's main social movements and civil society organizations find representation and political influence over the State through that party. But on the other, classical questions over cooptation and social movements autonomy return to the scene. At the same time, the opposition remains fluid and incapable of organizing stable and coherent parties, beind mostly reduced to some regionally located trenches. The aim of this article is to trace a map of the Bolivian political 
scene through an analysis of the main political themes and cleavages currently on the agenda after more than nine years of MAS government and how they relate to the incumbent party, the opposition and the main national social movements, as well as considerations over the primacy exerted by such movements over political intermediation in the country and their relationship with both incumbent and opposition parties.

Keywords: Bolivia; Political representation; Social movements; Processs of change; Movement towards socialism party.

\section{Introdución}

Bolivia es conocida por su tradicional inestabilidad política, hecho histórico demostrado al largo de su historia en una infinidad de golpes y contragolpes de Estado desde su fundación como República independiente en 1826 , pero que sin embargo parecía haber dejado en el pasado a partir de 1985. A partir de aquel año y hasta el 2002, el país logró una inédita transmisión constitucional ininterrumpida de cuatro mandatos presidenciales, su modelo institucional pasó a ser halagado como cauce de la estabilidad política (HOFMEISTER, 2004; MAYORGA, 1997) e importantes intelectuales se preguntaban si el país finalmente se encaminaba hacia la viabilidad democrática (CRABTREE; WHITEHEAD, 2001).

Sin embargo, pese a la estabilidad institucional lograda, el modelo daba ya señales de cansancio, con una creciente falta de identificación entre la población y los partidos hegemónicos, los cuales servían cada vez menos de canal institucional de mediación de intereses de la sociedad hacia el Estado (DOMINGO, 2001). De hecho con la llamada "Guerra del Agua" de Cochabamba en el año $2000^{1}$, la escalada del conflicto social trajo de vuelta las memorias no tan lejanas de inestabilidad política perene y la reedición de una posible historia sin fin de caos social (GUIMARÃES et al. 2009). De hecho, la siguiente "guerra" social, la del Gas en el año 2003, provocó no sólo la renuncia del entonces presidente Gonzalo Sánchez de Lozada, sino también el colapso del sistema partidario boliviano y el derrumbe del modelo institucional vigente, aunque no un quiebre de la democracia.

Bajo la aparente calma institucional anterior, una infinidad de movimientos sociales contra-hegemónicos de distintos tipos se movían en los subsuelos de la política boliviana (TAPIA, 2011b), logrando en los citados ciclos de rebelión social modificar sustancialmente a la política nacional. Ya las elecciones del 2002 habían dado al parlamento una cara literalmente distinta con el fuerte ingreso de diputados y senadores de

\footnotetext{
${ }^{1}$ Ver adelante.
}

origen indígena-campesina, pero son sobre todo las elecciones generales anticipadas del 2005, en las que por primera vez desde la democratización un presidente logra ser elegido en primera vuelta con mayoría absoluta, las que provocarán un radical cambio de élites políticas con la ascensión al poder del presidente Evo Morales y su partido Movimiento Al Socialismo - Instrumento Por la Soberanía de los Pueblos (MAS-IPSP o simplemente MAS). Un partido sui generis, que construye su identidad política a partir de la propia negación de su carácter de partido político, buscando presentarse como mero instrumento de los movimientos sociales y sindicatos para su acceso al y búsqueda de transformación del Estado boliviano, identificado por ellos como excluyente, colonial y poco democrático (HARNECKER; FUENTES, 2008; SILVA, 2009; ZUAZO, 2009).

Tras un primer mandato presidencial (2006-2009) en el cual la alta conflictividad con las oposiciones parecían dar razón a las hipótesis de inestabilidad política boliviana inmanente, Morales logra estabilizar su control institucional a partir de fines del 2008 (CUNHA FILHO, 2008), concluir el proceso constituyente con la aprobación de la nueva Constitución Política del Estado (en adelante, nCPE) en el 2009 y declarar la refundación del país de ahora en adelante llamado "Estado Plurinacional de Bolivia". Desde entonces, el presidente ha mantenido altos niveles de aprobación popular y ha avanzado la hegemonía del MAS sobre el sistema político boliviano, sin que las oposiciones consigan ofrecer un discurso programático alternativo que le pueda hacer frente (CUNHA FILHO, 2014).

Así, el presente texto buscará trazar un mapa de la política boliviana contemporánea, analizando los principales temas presentes en la agenda política actual $\mathrm{y}$ los actores que les encarnan, bien como analizar sus relaciones tanto con el campo oficialista como con el opositor. En la primera sección, se analizarán brevemente las razones que provocaron el derrumbe del sistema político vigente para de ahí pasar a un análisis de la polarización política vivida por el país entre el MAS y sus movimientos sociales aliados en búsqueda de la refundación institucional del país y las oposiciones 
regionales que buscaban enfrentarlos y cómo el campo oficialista logró superar dicha polarización. En seguida, el análisis buscará mostrar cómo, derrotada la oposición, las tensiones al interior del bloque oficialista se pusieron más evidentes y cuáles han sido sus consecuencias en términos de alineamientos de las organizaciones sociales con el partido oficialista y/o las oposiciones. En la tercera sección se analizará cómo el nuevo Estado Plurinacional parece estar firmándose como "sentido común de época" (ERREJÓN; CANELAS, 2012) y las implicaciones de eso sobre el conjunto de movimientos sociales y organizaciones políticas del país para, por último, concluir con un breve análisis de los resultados electorales del 2014 a la luz de lo expuesto en las secciones previas.

\section{Primer Momento: crisis y caída de la democracia pactada y la difícil génesis del nuevo país}

El período entre 1985-2002 quedó conocido como la "democracia pactada", debido al hecho de que ninguno de los partidos políticos lograra mayoría absoluta de los votos y, acorde a la Constitución del 1967 rescatada tras el final de la última dictadura militar, el presidente era elegido en balotaje indirecto por el parlamento en el cual los partidos pactaban acuerdos de gobierno y coalición. Dicho período estuvo enmarcado, además, por la aceptación por los principales partidos de las políticas de ajuste neoliberal, que no sólo no lograron solucionar los crónicos problemas de pobreza y desigualdad social bolivianos, sino más bien los agravaron (KOHL; FARTHING, 2006). Y en la medida en que todos los partidos relevantes habían confluido hacia una unanimidad en torno a la manutención de dichas políticas, crecía el descontento popular ante la falta de opciones programáticas entre los partidos tradicionales.

La coyuntura política empezó a cambiar a partir de la llamada "Guerra del Agua", cuando un grupo amplio de movimientos sociales agrupados bajo la Coordinadora por la Defensa del Agua y la Vida logró resistir al plan gubernamental del entonces presidente Hugo Bánzer de privatizar el sistema de agua de Cochabamba (VARGAS; KRUSE, 2000). A partir de ahí, una cantidad cada vez más grande de movimientos sociales y sindicatos que habían refluido a posiciones defensivas pasa a la ofensiva, con un aumento cada vez más grande de protestas y bloqueos por grupos indigenistas, sindicatos rurales, cocaleros y otros. Esto hasta que en el año 2003, ya bajo la segunda presidencia de Sánchez de Lozada, la propuesta presidencial de exportación del gas hacia
Estados Unidos y México, a través de puertos chilenos, catalizara una nueva oleada de protestas en la llamada "Guerra del Gas" tras la cual el presidente renuncia y huye del país ${ }^{2}$. Sin embargo, lo más transcendental de la "Guerra del Gas" fue la conformación de la llamada Agenda de Octubre ${ }^{3}$ centrada en la convocatoria para una Asamblea Constituyente (AC) en vistas a refundar el país y la nacionalización de los recursos hidrocarburíferos.

Cabe resaltar que en el período previo al derrumbe de la democracia pactada, los movimientos sociales del área rural agrupados sobre todo en la Confederación Sindical Única de Trabajadores Campesinos de Bolivia (CSTUCB) ${ }^{4}$, habían empezado a rediscutir la creación de un "instrumento político" que les sirviera de canal de inserción institucional en el Estado que buscaban transformar, todo eso en un fuerte contexto de recuperación de identidades indígenas. Asimismo, capitaneado sobre todo por los sindicatos cocaleros del Chapare, se funda el MAS-IPSP el cual disputaría con sigla propia por primera vez las elecciones del $2002^{5}$, para las que consiguió de manera sorpresiva el segundo lugar con $20,9 \%$, muy cercano a los $22,5 \%$ de Sánchez de Lozada quien sería finalmente escogido presidente por el Legislativo. Aunque no haya liderado directamente el ciclo de protestas del 2003, el MAS logra capitalizar las demandas de la Agenda de Octubre, presentándolas como suyas en las elecciones generales anticipadas del 2005, en las que obtiene inéditos $53,4 \%$ de los votos válidos conduciendo a la elección directa de Morales, sin necesidad de segunda vuelta congresal (ver Tabla 1).

Oficialmente, el MAS se autodefine como un instrumento de los sindicatos y organizaciones sociales que le dan base social y su núcleo central se encuentra en las Federaciones Cocaleras del Trópico de Cochabamba, desde el cual se amplía radialmente para incluir a la CSUTCB, la Confederación Sindical de Comunidades Interculturales de Bolivia (CSCIOB $)^{6}$ y la Confederación Nacional de Mujeres Campesinas Indígenas Originarias de Bolivia "Bartolina Sisa" (CNMCIOB-BS). Desde ese núcleo duro, el partido

\footnotetext{
${ }^{2}$ Véase Hylton y Thomson (2007).

3 En referencia al mes en que ocurrieron las principales confrontaciones $\mathrm{y}$ en el que renuncia el presidente.

${ }^{4}$ Confederación de los sindicatos rurales bolivianos de alcance nacional.

5 Por dificultades burocráticas con el registro de la organización que originalmente se llamaría Asamblea por la Soberanía de los Pueblos, lo que posteriormente se convertiría en MAS participó de las elecciones del 1997 bajo invitación del partido Izquierda Unida. En estas elecciones, Morales logra su primera elección a diputado nacional (HARNECKER; FUENTES, 2008; ZUAZO, 2009).

${ }^{6}$ Anteriormente llamada Confederación Sindical de Colonizadores de Bolivia.
} 
Tabla 1. Resultados Electorales Post-Democratización, República de Bolivia.

\begin{tabular}{lccccccc}
\hline & $\mathbf{1 9 8 0}$ & $\mathbf{1 9 8 5}$ & $\mathbf{1 9 8 9}$ & $\mathbf{1 9 9 3}$ & $\mathbf{1 9 9 7}$ & $\mathbf{2 0 0 2}$ & $\mathbf{2 0 0 5}$ \\
\hline Candidato & Hernán Siles & Victor Paz & Jaime Paz & Sánchez & Hugo & Sánchez & Evo \\
Elegido & Zuazo & Estenssoro & Zamora & de Lozada & Bánzer & de Lozada & Morales \\
(Partido) & $(\mathrm{UDP})$ & $(\mathrm{MNR})^{\mathrm{a}}$ & $(\mathrm{MIR})^{\mathrm{b}}$ & $(\mathrm{MNR})$ & $(\mathrm{ADN})$ & $(\mathrm{MNR})$ & $(\mathrm{MAS})$ \\
Voto Nacional & $38,74 \%$ & $30,36 \%$ & $21,82 \%$ & $35,55 \%$ & $22,26 \%$ & $22,46 \%$ & $53,74 \%$ \\
\hline
\end{tabular}

Fuente: Elaboración propia con datos disponibles en Mesa Gisbert (2006). *Debido al golpe militar de Luis García Meza, el presidente elegido solamente asumiría en 1982. ${ }^{\mathrm{a}}$ Segundo lugar en el sufragio popular. ${ }^{\mathrm{b}} \mathrm{Tercer}$ lugar en el sufragio popular.

ha buscado atraer a sus filas, con mayor o menor organicidad, a otros sectores gremiales como la Federación Nacional de Cooperativas Mineras (Fencomin) y sindicatos urbanos. Además de eso, entre los años 2002-2004, las principales organizaciones rurales del país entre las cuales se incluyen a todas las organizaciones base del MAS (con excepción de los cocaleros $^{7}$ ) más la Confederación Indígena del Oriente Boliviano (CIDOB $)^{8}$ y el Consejo Nacional de Marqas y Ayllus del Qollasuyu (CONAMAQ) ${ }^{9}$, gestaron el llamado Pacto de Unidad, a través del cual buscaban concertar posiciones comunes hacia un proyecto de nueva constitución capaz de dar cuenta de la descolonización del Estado a partir de las demandas indígena campesinas. El Pacto de Unidad ha sido la principal base de apoyo al primer gobierno de Morales y el actor fundamental de la Asamblea Constituyente (AC) convocada entre agosto de 2006 y diciembre de 2007 (GARCÉS 2012, 2013; SCHAVELZON, 2012).

Mientras tanto, las élites políticas tradicionales desplazadas del poder entre 2003-2005 buscaron confrontar a la Agenda de Octubre demandas de descentralización administrativa y autonomía departamental, en lo que quedó conocido como la "Agenda de Enero", por la realización en Enero de 2005 de un cabildo en Santa Cruz de la Sierra con la demanda de elección directa de los entonces llamados prefectos departamentales. Dicha demanda fue aceptada como parte de los acuerdos para la realización de las elecciones anticipadas de diciembre de 2005, en las cuales por primera vez se votó para la elección de los prefectos anteriormente designados directamente por el presidente. Y tras la victoria de grupos opositores conformados a partir de los fragmentos de los anteriormente hegemónicos

\footnotetext{
${ }^{7}$ Los cuales, sin embargo, están afiliados a la CSUTCB, por lo cual se puede considerar que hayan participado indirectamente.

${ }^{8}$ Organización que agrupa a los pueblos indígenas de la Amazonía, Chaco y tierras bajas bolivianas.

${ }^{9}$ Organización que agrupa a las comunidades indígenas del Altiplano boliviano que rechazan la forma organizativa sindical - y por ende la afiliación a la CSTUCB - y buscan reconstruir formas tradicionales de mando comunal.
}

partidos tradicionales en los departamentos de Pando, Beni, Santa Cruz y Tarija (que pasaron a ser conocidos como la Media Luna, por el formato en arco de dichos departamentos del Norte, Oriente y Sur en el mapa boliviano), la oposición política al MAS buscó articular un potente movimiento regionalista de demandas por mayor autonomía departamental. Al principio tajantemente rechazado por el presidente Morales como un movimiento de élites aristocráticas que buscaba dividir a Bolivia, los prefectos de la Media Luna lograron, sin embargo, una fuerte aceptación popular entre sectores urbanos de sus regiones al activar clivajes e identidades regionales realmente existentes (ERREJÓN; CANELAS, 2012; CLAROS, 2010), y que parecían contraponer al altiplano occidental del país a sus planicies orientales. El embate entre gobierno y movimientos sociales afines y el movimiento regional liderado por los prefectos de la Media Luna prácticamente acaparó a la política boliviana entre 2007 y 2008 y casi hace naufragar a la AC. Ésta solamente lograría concretarse tras una concertación entre el gobierno y sectores de la oposición más independientes de los prefectos de la Media Luna, que en una comisión conjunta en el Legislativo harían una serie de revisiones al proyecto constitucional aprobado por la AC y finalmente convocarían al referendo ratificatorio al texto de la nCPE, la cual sería aprobada en enero de 2009 (CUNHA FILHO, 2008; SCHAVELZON, 2012).

La conclusión del proceso constituyente trajo consigo, además, un debilitamiento del movimiento opositor regionalista, en la medida en que sus sectores más radicales perdieron mucho de su legitimidad por acciones violentas en septiembre de 2008 y, sobre todo, por el hecho de que la nCPE concertada en el Legislativo logró capturar a la demanda autonomista departamental y hacer de ella un componente central del nuevo modelo de Estado Plurinacional abanderado por el gobierno (ERREJÓN; CANELAS, 2012, p. 27). Lo que quedó entre el referéndum constitucional de enero y las elecciones nacionales de diciembre de 2009 podría ser descrito como un período de notable calma institucional en espera de la cierta reelección presidencial de Evo Morales, la cual efectivamente 
se daría con el $64,22 \%$ de los votos válidos. Pero tal descripción encubriría mucho de las tensiones latentes entre el MAS y partes importantes de su base social, las cuales ya se manifestaban en alguna medida en la preparación previa al proceso constituyente, en el interior de la $\mathrm{AC}$ y nuevamente durante lo bastante tranquilo año político de 2009.

\section{Segundo Momento: las pugnas internas a la implementación del Estado Plurinacional y las tensiones entre el MAS y movimientos sociales}

Aunque el debilitamiento de las oposiciones, arrinconadas en sus regiones y sin capacidad de enfrentamiento al gobierno central como opción programática nacional, podría hacernos creer en una probable disminución de eventos de protesta social, la verdad ha sido todo lo contrario. En número de eventos de protesta callejera, por ejemplo, el año 2011 ha sido el más conflictivo de los 42 años anteriores en el país con un total de 1300 protestas registradas al largo del país (MARTÍ I PUIG; BASTIDAS, 2012; FONTANA, 2013; CRESPO; MAYORGA, 2012). De esta forma, ¿qué podría explicar semejante paradoja?

Es necesario considerar que el MAS, en su relación con los movimientos sociales, sindicatos y organizaciones populares es, y siempre ha sido, una especie de coalición inestable cuyos términos son renegociados a cada rato, dependiendo de la coyuntura (MAYORGA, 2011; SILVA, 2009). Durante su primer mandato presidencial, pero sobretodo en el período que va de mediados del 2006 hacia septiembre-octubre del 2008, la fuerza política demostrada por la oposición anclada en sus movimientos regionalistas de base amplia, hacía que la defensa del gobierno "de los movimientos sociales" frente a lo que podrían ser intentos golpistas o separatistas fuese la prioridad número uno ante cualquier divergencia programática entre los diversos miembros de su base social.

Pero aún en ese momento, existieron tensiones importantes al interior de esa base. Durante los trabajos de la AC, por ejemplo, eran notables los esfuerzos para encontrar un mínimo denominador común a las propuestas de las distintas organizaciones que conformaron al Pacto de Unidad (SCHAVELZON, 2012; SCHILLING-VACAFLOR, 2008), los cuales se lograban no sin tensiones visibles y que, en ciertas ocasiones, inviabilizaron los pactos internos ${ }^{10}$. Y aún antes de la inauguración del proceso constituyente, hubo

\footnotetext{
${ }^{10}$ El CONAMAQ, por ejemplo, llegó a declararse fuera del Pacto en determinado momento durante los trabajos de la AC (véase Schavelzon, 2012).
}

críticas importantes de movimientos y organizaciones sociales a la ley de convocatoria a la AC por mantener un sistema de elección de constituyentes a través de partidos cuándo ellos demandaban algún tipo de sistema alternativo de nombramiento autónomo a partir de las propias organizaciones y movimientos sociales (CHÁVEZ; MOKRANI, 2007; GUTIÉRREZ AGUILAR, 2008).

Tras la aprobación de la nCPE en el referéndum de enero de 2009, dichas tensiones se pusieron aún más visibles en los debates para la aprobación de algunas de las leyes de reglamentación del texto constitucional. Sobre todo para la aprobación de la Ley Electoral Transitoria (LET), la cual reglamentaria los procedimientos electorales de fines de 2009 para la renovación del Parlamento y presidencia del Estado Plurinacional, CIDOB y CONAMAQ demostraron públicamente su descontento con el número de escaños especiales indígenas en la propuesta oficialista: el proyecto enviado por el gobierno al Parlamento reservaba 14 escaños a los indígenas de etnias minoritarias, mientras CONAMAQ demandaba 18 y CIDOB 24. Al final, por las resistencias opositoras en el Senado, en la LET aprobada quedaron apenas 7 escaños especiales indígenas (uno por departamento con excepción de Potosí y Chuquisaca) y aunque molestas, ambas organizaciones indigenistas aceptaron el resultado ${ }^{11}$.

Pero tras la fácil reelección de Morales y el inicio de su segundo mandato ya bajo la nCPE y con sólida mayoría en las dos casas legislativas en 2010, además de la conquista de la mayoría de las gobernaciones departamentales y alcaldías en las elecciones de abril de 2010 (CUNHA FILHO, 2010), se incrementa el número de protestas de grupos variados, la mayoría de ellos apoyadores del 'Proceso de Cambio'. Aunque algunas protestas necesitaron ser solucionadas mediante una mezcla de represión violenta y soluciones salomónicas, como el conflicto en Caranavi por la ubicación de una planta procesadora de cítricos en abril-mayo de $2010^{12}$, o llegaron a alcanzar grados importantes de magnitud, como la protesta regional en Potosí que paralizó el departamento por 19 días en demanda por recursos y políticas públicas específicas en agosto de

\footnotetext{
${ }^{11}$ Lo que, sin embargo, no es poco si se consideran la proporción entre ese número y el total de diputados ( 7 sobre 130 , poco más del $5 \%$ del total) y entre la población total de las etnias minoritarias y de Bolivia, la cual según Diego Ayo (2010, p. 125) es de aproximadamente 4,5\%.

${ }^{12}$ Los ciudadanos de Caranavi protestaban contra la instalación de dicha planta en la región de Alto Beni y reclamaban dicha planta para su localidad. La represión policial para liberar la ruta bloqueada por los pobladores en protesta dejó un saldo de dos muertos y alrededor de 40 heridos, después de la cual una negociación entre líderes de la protesta y el gobierno decidió por la instalación de dos plantas procesadores de cítricos, una en cada localidad (OBSERVATÓRIO POLÍTICO SUL-AMERICANO, 2014).
} 
$2010^{13}$; éstas no consistieron en protestas en contra del gobierno buscando desestabilizarlo, sino más bien que expresaban la erupción de demandas - a veces contradictorias entre si - que el sociólogo y vicepresidente Álvaro García Linera (2011) buscaría teorizar como la emergencia de tensiones creativas al interior del 'Proceso de Cambio'.

La primera crisis verdadera entre el gobierno y su base de apoyo vendría en fines de 2010, cuando el 26 de diciembre el gobierno emitió un decreto suspendiendo la subvención a los combustibles cuyos precios se encontraban congelados desde hacía años, y que en la práctica representó un alza de precios de entre el $57 \%$ y el $82 \%$ (dependiendo del tipo de carburante), quedando conocida como el "Gasolinazo". Pese al anuncio concomitante de medidas destinadas a mitigar el impacto de dicha alza - como aumentos salariales, la conversión gratuita de vehículos al uso de GNV etc. - el resultado fue una masiva protesta social que paralizó al país por días hasta que en vísperas del año nuevo el presidente Morales anunciara la suspensión de la medida y el retorno al régimen de subvención estatal a los combustibles. Aunque lamentando el enorme costo económico que dicha subvención representaba al Estado, el presidente buscó enmarcar la vuelta atrás como demostración de un gobierno abierto a escuchar la voz de las calles y hacer lo que el pueblo demandaba.

El "Gasolinazo" fue un momento crítico del cual los opositores buscaron sacar réditos políticos criticando al gobierno de haber tomado una medida neoliberal como las que tanto había criticado en su camino a la presidencia, pero aunque al principio haya impactado los niveles de aprobación del gobierno ante la opinión pública, no tuvo mayores consecuencias políticas posteriores. Pero la siguiente crisis, provocada por el proyecto de construcción de una carretera conectando Villa Tunari (Cochabamba) a San Ignacio de Moxos (Beni), sí alcanzó una importancia simbólica más grande y para muchos representó un verdadero parteaguas del gobierno (ROSSELL, 2012; ORTIZ, 2011).

Dicha carretera atravesaría el corazón del Territorio Indígena y Parque Nacional Isiboro-Sécure (TIPNIS) y las obras de su construcción así como la obtención de recursos para su financiamiento se habían dado sin la ejecución de una consulta previa e informada con los indígenas afectados, tal como demandaba la

\footnotetext{
${ }^{13}$ Convocada por el Comité Cívico de Potosí (COMCIPO), la protesta demandaba inicialmente la demarcación de límites territoriales entre las localidades de Coroma (Potosí) y Quillacas (Oruro), región rica en yacimientos de piedra caliza y cuyos límites departamentales eran imprecisos. Pronto la protesta escaló hacia la demanda de construcción y mejoramiento de carreteras, la reactivación del complejo polimetalúrgico de Karachipampa y la construcción de un aeropuerto internacional en la capital departamental y de una fábrica cementera en la región (OBSERVATÓRIO POLÍTICO SUL-AMERICANO, 16/08/2010).
}

nCPE. En protesta por la construcción inconsulta de la carretera, la CIDOB convocó a una marcha desde Trinidad (Beni) hacia La Paz, la cual partió el 15 de agosto de 2011. Mientras los indígenas marchaban, un sindicato campesino de Yucumo (Beni) anunció que bloquearía el paso de la marcha. El gobierno intentó por dos veces enviar comisiones de ministros para negociar con los indígenas sin éxito en la suspensión de la marcha, y cuando la misma ya se acercaba a Yucumo, envió al canciller David Choquehuanca a negociar otra vez e intentar evitar el confronto inminente entre los marchistas y el sindicato. Sin embargo el canciller terminó cayendo como rehén y escudo humano por algunas horas en el camino hacia Yucumo, con lo cual el gobierno ordenó la dispersión de la marcha, represión policial mediante, el 25 de septiembre.

El episodio todavía queda irresuelto en lo que concierne a la cadena de mando de la orden de represión. La entonces ministra de Defensa, Maria Cecília Chacón, renunció por no haber sido comunicada de la decisión. La posición oficial del gobierno, hasta el momento, consiste en que el presidente Morales no habría ordenado la represión a los marchistas, que ésta había sido ordenada sin consulta a los superiores por el entonces viceministro de Régimen Interior, Marcos Farfán. Tras el frustrado intento de dispersión de la marcha, ésta arribó a La Paz el 19 de octubre y representantes de los marchistas se reunieron con el presidente Morales, obteniendo la promesa de que no se construiría la carretera, la cual se formalizaría en la Ley 180 el 24 de octubre.

Pero el 20 de diciembre otra marcha partió de la región del TIPNIS, esa vez organizada por el Consejo Indígena del Sur (Conisur), liderado por el cacique Gumercindo Pradel demandando al gobierno, por el contrario, la retomada de la construcción de la carretera. Dicha marcha arribó a La Paz el 30 de enero de 2012 y obtuvo del gobierno la aprobación el 10 de febrero de la Ley 222 de Consulta a los Pueblos del TIPNIS, para determinar si se construiría o no la carretera. La CIDOB, organizadora de la primera marcha, acusó al Conisur de haber sido cooptado por el gobierno y rechazó la realización de la consulta, organizando una nueva marcha que partió de Trinidad el 27 de abril y llegó a La Paz el 27 de junio. Sin embargo, esta vez no fueron recibidos por el presidente y dejaron la ciudad el 10 de julio sin éxito. Mientras tanto, sectores disidentes de la CIDOB convocaron a una asamblea en la ciudad de Santa Cruz de la Sierra y destituyeron al anterior líder y organizador de las marchas, Adolfo Chávez, remplazándolo por Melva Hurtado. Esta decisión fue rechazada por el grupo dirigido por Chávez, el cual alegó ilegalidades en la asamblea. Por ende, en la actualidad coexisten dos CIDOBs paralelas: una opositora y otra afín al gobierno. 
La consulta a los pueblos del TIPNIS convocada por la Ley 222 empezó el 29 de julio y finalizó en diciembre de 2012, con sus resultados hechos públicos el 07 de ese mes por el ministro de Obras Públicas, Vladimir Sánchez. Fueron consultadas 58 comunidades de las 69 existentes en el TIPNIS, debido a que 11 de ellas (afines a la CIDOB opositora) rechazaron participar del proceso y prohibieron el ingreso de los funcionarios del Órgano Electoral. De acuerdo al informe final detallado difundido en 07 de enero de 2012, de las comunidades consultadas 54 se habrían manifestado favorables a la construcción de la carretera, que en la actualidad continúa congelada y sigue siendo un tema de controversias en la sociedad boliviana.

La importancia del conflicto reside en que arañó la imagen internacional de Morales como "defensor de la Pachamama" difundida por el propio gobierno, bien como por representar un quiebre del Pacto de Unidad, con una separación entre entidades rurales del núcleo duro de apoyo al gobierno, como CSUTCB, Bartolinas e Interculturales, y organizaciones indianistas como CIDOB y CONAMAQ las cuales pasaron, al menos en parte, a hacerle oposición. El conflicto trajo además la vuelta de un cuestionamiento sobre quienes serían verdaderamente indígenas y quienes serían meramente campesinos, debate insoluble y poco productivo en la medida en que la mayoría de los actores rurales son ambas cosas al mismo tiempo (ALBÓ, 2010), por no hablar del hecho de que la mayoría de la población boliviana ya es urbana, incluidos sus indígenas, lo que seguramente influye en las consecuencias prácticas de sus identificaciones personales.

Sin embargo, el conflicto del TIPNIS reactivó también, y ahora con más fuerza, críticas por parte de grupos de intelectuales y movimientos sociales más autonomistas y antes afines al gobierno a lo que califican como una visión hegemonista del MAS sobre el conjunto de la pluralidad boliviana (MOKRANI; URIONA CRESPO, 2011; TAPIA, 2011a). Como anteriormente mencionado, el MAS se considera a sí mismo como un instrumento de los movimientos sociales, rechazando la imagen de partido político, y al cual se accede como militante a partir de la membresía a alguna de las organizaciones sociales de su base. Esto, aunque en el camino hacia su primera elección en 2005 y especialmente tras su llegada al gobierno dicho principio haya sido relativizado con la presencia cada vez mayor de la figura de los "invitados", personalidades no originalmente militantes del partido a quien se le reserva alguna candidatura importante y cuyo principal ejemplo es el vicepresidente García Linera. Pero el grueso de su militancia todavía adviene de organizaciones sociales de base sindicales, indígenas, vecinales y muchas otras.
Sin embargo, es cierto que en el gobierno, pese a que siga declarándose como el "gobierno de los movimientos sociales" - y así sea reconocido internacionalmente por muchos intelectuales (por ejemplo, QUIROGA, 2010) - el MAS sí viene buscando supeditar los conflictos sociales a las "razones de Estado" conforme determinadas por el programa de desarrollo y los objetivos políticos del gobierno. Como muestra Molina (2013, p. 12), el tratamiento dado por el gobierno a las movilizaciones sociales varía conforme a cómo los percibe sea como enemigos o aliados. En caso en que sean considerados como "aliados", aparece llamativo que el gobierno haya buscado, de forma generalizada, en encuadrarlas como "tensiones creativas del proceso de cambio" (Linera, 2011) tratando además de desactivarlas recurriendo a las relaciones con sus dirigentes y a concesiones puntuales. De forma opuesta, si son consideradas adversarias, o si las movilizaciones de entes considerados aliados sobrepasan los límites “tolerables", el gobierno busca desacreditar al movimiento e incluso a veces moviliza otros sectores más leales en contra de los movilizados. Y si bien ha logrado hasta aquí encuadrar a la parte más importante de las protestas como tensiones creativas del proceso, ha generado también desgastes y disidencias importantes tanto con organizaciones sociales, como con miembros del propio partido y/o gobierno que se han alejado y buscado otras opciones partidistas.

\section{Tercer momento: ¿consolidación del sentido común de época?}

Pero lo más políticamente relevante en los momentos de fuerte movilización social del segundo periodo de gobierno del MAS parece ser la consolidación de términos del embate político dentro de los marcos del nuevo "sentido común de época", tal como definido por el nuevo Estado Plurinacional (ERREJÓN; CANELAS, 2012, p. 28): tanto en el "gasolinazo" cuanto en el conflicto por el TIPNIS, por ejemplo, los términos del debate y de las críticas hacia el gobierno y el partido oficialista iban en el sentido de que el gobierno supuestamente incumplía los objetivos a que se había propuesto con la nueva CPE y su plan de desarrollo. Las críticas al "gasolinazo" como una medida neoliberal advenida de sectores hasta ayer defensores de tal modelo implican, aunque quizás inconscientemente, una aceptación de los nuevos tiempos económicos en que el Estado ha vuelto a ser un actor fundamental en la organización de la economía boliviana. Del mismo modo, las críticas a la carretera por el TIPNIS se centraron principalmente en la tentativa inicial de evadir a los procedimientos de consulta previa a las comunidades indígenas locales y 
en la supuesta violación de la libre determinación de dichas comunidades conforme establecidos en la nCPE.

Eso tiene una importancia transcendental ya que resta a las oposiciones mucho de las posibilidades de construcción de un discurso programático alternativo capaz de hacer frente al gobierno. De hecho, ante esa falta de un discurso programático unificador, los partidos opositores ubicados a la derecha del espectro político, han buscado reciclarse con el uso de símbolos y nombres que resuenan a una ideología de izquierda ${ }^{14}$, como el Movimiento Demócrata Social (MDS) liderado por el gobernador de Santa Cruz, Rubén Costas, o la tentativa de construcción del Frente Amplio por el empresario cementero Samuel Doria Medina, los cuales terminaron por conformar una alianza electoral bautizada Concertación Unidad Demócrata (UD) con Doria Medina como candidato a la presidencia y el exgobernador del Beni, Ernesto Suárez, como candidato a vicepresidente. Pero dicha alianza no se dio entre MDS y el Frente Amplio, sino con el partido original de Doria Medina, Unidad Nacional (UN), lo que terminó por implosionar al Frente Amplio, tras la deserción de la mayor parte de su ala izquierda y el abandono del proyecto que se promocionaba como un espacio de encuentro y debates programáticos hacia más allá del horizonte electoral del 2014.

Por el otro lado, partidos centro-izquierdistas como el ex aliado Movimiento Sin Miedo (MSM) ${ }^{15}$, pese a haber buscado una alianza con el derechista MDS que parecía encaminada a concretarse hasta muy poco antes de la conformación de UD, han buscado, con cierto éxito, a atraer a disidentes del MAS. Sin embargo solo consiguieron atraer algunas personalidades, aunque relevantes, no consiguiendo convencer a movimientos $\mathrm{u}$ organizaciones sociales en bloque. Muchos ex integrantes importantes del partido oficialista, como la ex presidenta de la Cámara de Diputados (2012-2013), Rebeca Delgado, se han ido sumando al MSM, pero no así ninguna organización social de relevancia. Esto

\footnotetext{
${ }^{14}$ La única excepción fue el expresidente Jorge “Tuto" Quiroga (2001-2), quien se lanzó a la presidencia por el Partido Demócrata Cristiano (PDC) y buscó asociar su imagen a una oposición conservadora a Evo y el MAS, con alusiones explícitas a la fe católica y promesas de endurecimiento en el combate a los cultivos ilegales de coca - pese a que el área total de coca cultivada en Bolivia haya más bien sido reducida durante los gobiernos de Morales y con disminución de la conflictividad social en el rubro (véase FARTHING; KOHL, 2010).

${ }^{15}$ El MSM fue aliado formal del MAS entre 2005 y 2010, habiendo presentado sus candidatos a diputados en las listas electorales del MAS en las elecciones generales del 2005 y 2009. La alianza fue una de las estrategias del MAS para penetrar el voto urbano donde tenía mayor dificultad y el MSM, con agenda programática semejante, pero de base social predominantemente clasemediera y controlando la alcaldía de La Paz desde 1999, resultó bastante cómoda. Sin embargo, para las elecciones regionales departamentales y municipales del 2010, los dos partidos rompen la alianza por disputas en torno a candidaturas (véase CUNHA FILHO, 2010)
}

se debe, en parte, a que organizaciones que han tenido serios enfrentamientos con el gobierno, como CIDOB y CONAMAQ, se han escindido en alas oficialistas y opositoras, en vez de formar bloques homogéneos susceptibles de pasar a la oposición. Y aún con las fracciones opositoras, no se ha dado una migración en bloque hacia ninguna sigla partidista, sino más bien un fraccionamiento entre sus líderes quienes han optado por distintas alternativas: por ejemplo, el diputado por la representación indígena del Beni, Pedro Nuny, elegido por el MAS para la legislatura 2010-2014, se ha aliado al MDS en la gobernación de su departamento; uno de los líderes del ala opositor del CONAMAQ, Rafael Quispe, se sumó al intento de conformación del Frente Amplio y tras la disolución del mismo, se ha quedado como candidato a diputado en la alianza UD.

Asimismo, dirigentes sociales que han buscado construir en bloque una alternativa nacional al MAS han sido poco exitosos. El dirigente de una de las subcentrales del TIPNIS, Fernando Vargas, rechazó invitaciones a sumarse al MSM y se postuló a la presidencia por el recién creado Partido Verde Boliviano (PVB), con un discurso fuertemente indianista y ambientalista, pero que ha generado poco entusiasmo, al conseguir siempre el último lugar en las encuestas electorales. También la histórica Central Obrera Boliviana (COB), la cual tradicionalmente ha tenido entre sus dogmas la independencia obrera y venía criticando fuertemente al gobierno desde el gasolinazo, y cuya dirigencia venía gestando la creación de una sigla propia - el Partido de los Trabajadores - decidió en 2014 abandonar dicho proyecto y sumarse a las filas oficialistas indicando candidatos a diputados en las listas del MAS.

Y ante la perspectiva de fácil reelección presidencial de Morales, incluso muchos disidentes que se habían sumado a otros partidos, como el ex ministro del Agua Abel Mamani, empezaron a volver a las filas del partido oficialista, incluso muchos que constaban como candidatos a diputado por sus partidos y al volver al MAS han quedado fuera de las listas electorales del 2014.

\section{Consideraciones finales}

La abrumadora reelección de Evo Morales el 12 de octubre de 2014, con una votación apenas poco debajo de la obtenida cinco años antes $(61,36 \%$ de los votos válidos), no ha sido en sí misma una sorpresa y confirma la hegemonía política lograda por el MAS: además de la elevada votación nacional, en la votación por departamentos el partido ha logrado por primera vez ser el más votado en el antiguo bastión opositor de Santa Cruz (aunque todavía sin mayoría absoluta) y 
sólo en el Beni ha llegado en segundo lugar con $41,49 \%$ contra 51,44\% de UD (ver Tabla 2). Pero sí ha habido sorpresas y también confirmaciones otras que ponen interesantes interrogaciones sobre los futuros políticos del país y los realineamientos sociales y electorales.

La primera tiene que ver con las sorpresivas votaciones del PVB y del MSM. En el primer caso, sorpresiva en un sentido positivo a las aspiraciones del partido al obtener $2,65 \%$, lo que le habría garantizado la elección de un diputado en la lista plurinominal vinculada a la votación presidencial. Y el segundo caso, del MSM, una sorpresa negativa ya que el partido - guardadas las debidas proporciones, el único junto al MAS con efectiva presencia nacional (CUNHA FILHO, 2010) - con apenas 2,71\% y también apenas un diputado potencialmente elegido esperaba mejor cosecha electoral entre los electores con un perfil a la izquierda del centro que se habían alejado de Morales. Al parecer, el PVB ha logrado atraer justamente a una parte de ese electorado y sobre todo en su bastión paceño. Pero vale resaltar que ambos deberán volver a tramitar sus personerías jurídicas ya que las leyes electorales bolivianas establecen la pérdida de las mismas a partidos que no obtengan un mínimo de $3 \%$ de los votos nacionales y esto implica la pérdida de los diputados que habrían elegido. Lo que harán políticamente en los próximos años y especialmente como se portarán uno frente al otro en ese proceso de reconstrucción partidaria podrá ser determinante para las posibilidades de aglutinación de sectores descontentos con el gobierno desde una visión

Tabla 2. Voto por el MAS, Estado Plurinacional de Bolivia.

\begin{tabular}{lcc}
\hline & \multicolumn{2}{c}{$\%$ de los votos válidos } \\
\hline Nacional y Exterior & 2009 & 2014 \\
& 64,22 & 61,36
\end{tabular}

Por departamentos

\begin{tabular}{lll} 
La Paz & $\mathbf{8 0 , 2 8}$ & $\mathbf{6 8 , 9 2}$ \\
Oruro & $\mathbf{7 9 , 4 6}$ & $\mathbf{6 6 , 4 2}$ \\
Potosí & $\mathbf{7 8 , 3 2}$ & $\mathbf{6 9 , 4 9}$ \\
Cochabamba & $\mathbf{6 8 , 8 2}$ & $\mathbf{6 6 , 6 7}$ \\
Chuquisaca & $\mathbf{5 6 , 0 5}$ & $\mathbf{6 3 , 3 8}$ \\
Tarija & $\mathbf{5 1 , 0 9}$ & $\mathbf{5 1 , 6 8}$ \\
Santa Cruz & $40,91 \%$ & $\mathbf{4 8 , 4 9 \%}$ \\
Pando & $44,51 \%$ & $\mathbf{5 2 , 0 9 \%}$ \\
Beni & $37,66 \%$ & $41,49 \%$ \\
\hline
\end{tabular}

Negritas indican primera mayoría. Fuente: Elaboración propia a partir de datos del Órgano Electoral Plurinacional (BOLIVIA, 2014). izquierdista o de autonomismo indianista, esta vez incorporando orgánicamente a sectores y agrupaciones (o por lo menos sus fracciones públicas) y no sólo a personalidades.

La segunda no es propiamente una sorpresa, sino una novedad: por primera vez, el adversario al MAS más bien votado no ha venido de la oposición más dura y conservadora como en las anteriores dos veces (Tuto Quiroga en 2005 y Manfred Reyes Villa en 2009), sino de su sector más blando y que, como mencionado, venía buscando ubicarse con simbologías de izquierda y un discurso de oposición propositiva representado por Samuel Doria Medina (UD), con sus $24,23 \%$, frente a los 9,04\% de Tuto Quiroga (PDC), quien sí buscó ubicarse en esa posición. Doria Medina, quien había sido candidato por $\mathrm{UN}$ en las dos elecciones anteriores también con un discurso de oposición moderada, había sido precisamente la "víctima" de la extrema polarización electoral boliviana y quedado en tercer lugar por no representar el campeón anti-Evo radical que llevara Quiroga y Reyes Villa al segundo puesto anteriormente (MOLINA, 2010). El resultado actual puede muy bien significar, como analiza Fernando Molina (2014), el fin de la polarización electoral en el país, pero hay que recordar que UD es una coalición electoral entre UN y MDS, cuyos líderes (incluido el candidato vicepresidencial de UD) hasta hace muy poco personificaban precisamente a esa oposición radical anti-Evo. En qué medida UD podrá ubicarse como una oposición constructiva como prometió Doria Medina al reconocer su derrota o si va a fraccionarse entre sus dos partidos núcleo determinará bastante el cuánto ese fin de la polarización podrá ser duradero, resultado que también dependerá en parte de cómo Tuto Quiroga logrará (o no) capitalizar su no despreciable capital político conquistado en esas elecciones cuándo ya parecía retirado de la escena política nacional. Pero al parecer, finalmente se va reconstruyendo un sistema partidario mínimamente coherente en Bolivia tras la implosión de la democracia pactada, con un polo hegemonizado a la izquierda por el MAS, una centro-derecha y una derecha más conservadora representados por UD y PDC respectivamente y fracciones de centro-izquierda e indianismo autonomista minoritarias y en búsqueda de consolidación de un mecanismo electoral.

Finalmente, habrá que observar de qué forma la expansión geográfica del MAS, ahora también líder electoral en Santa Cruz, incrementará sus tensiones internas y en qué medida el partido será capaz de procesarlas adecuadamente. Del mismo modo que la "conquista de Pando"16 (DO ALTO; STEFANONI,

\footnotetext{
${ }^{16}$ La conquista de su gobierno departamental en el 2010, ya que como visto en la Tabla 2, también apenas en el 2014 el MAS logra la mayoría de los votos presidenciales en el departamento.
} 
2010), la victoria en Santa Cruz ha implicado una incorporación o aproximación del partido oficialista con sectores antes reacios a su presencia, como el empresariado, o la incorporación de antiguos liderazgos reciclados de los extintos partidos tradicionales que no fue exenta de tensiones internas y bien puede ser la explicación de la baja relativa de votos del MAS en sus bastiones altiplánicos.

Si bien es cierto que el MAS realmente tiene una visión "hegemonista" de que la representación de los movimientos sociales debe darse necesariamente a través de sus canales internos como apuntado por Mokrani; Uriona Crespo (2011), también es cierto que es ya parte de la cultura política de dichas organizaciones buscar acceso a mecanismos de poder estatal a través de su incorporación a organizaciones políticas y que tras dichos canales el MAS ha logrado incorporar al juego democrático a amplias camadas $\mathrm{y}$ grupos sociales anteriormente excluidos. $\mathrm{Y}$ tal como descrito por Fernando Mayorga (2011), dicho partido es y siempre ha sido una coalición inestable de organizaciones y movimientos sociales. Dicha coalición necesita ser frecuentemente renegociada como forma de mantener su cohesión y eso limita considerablemente las posibilidades de cooptación definitiva de las organizaciones sociales por parte del partido, así que el incremento de intereses agregados a la coalición y que necesitarán ser balanceados seguramente complejizará el mantenimiento de su cohesión.

\section{Referencias}

ALBÓ, X. Lo indígena originario campesino en la nueva Constitución. In: Vicepresidencia del Estado Plurinacional De Bolivia; IDEA Internacional; Umsa. (Org.). Miradas: nuevo texto constitucional. La Paz: Universidad Mayor de San Andrés, 2010. p. 713-724.

AYO, D. S. Las autonomías entre "hegemonistas y consociativos". In: Vicepresidencia del Estado Plurinacional De Bolivia; IDEA Internacional; Umsa. (Org.). Miradas: nuevo texto constitucional. La Paz: Universidad Mayor de San Andrés, 2010. p. 165-178.

BOLIVIA. Tribunal Supremo Electoral. La Paz: Tribunal Supremo Electoral, XXXX. Disponible en: $<\mathrm{http}: / /$ www.oep.org.bo/>. Acceso en: 15 dec. 2014.

CHÁVEZ, P.; MOKRANI, D. Los movimientos sociales en la Asamblea Constituyente. Hacia la reconfiguración de la política. In: SVAMPA, M.; STEFANONI, P. (Org.). Bolivia: memoria, insurgencia y movimientos sociales. Buenos Aires: Editorial El Colectivo, 2007. p. 55-66.

CLAROS, C. P. Un pueblo eminente: autonomist populism in Santa Cruz. Latin American Perspectives, v. 37, n. 4, p. 125-139, 2010.
CRABTREE, J.; WHITEHEAD, L. Towards democratic viability: the Bolivian experience. Basingstoke: Palgrave Macmillan, 2001.

CRESPO, S. O.; MAYORGA, F. Movimientos sociales, Estado y democracia en Bolivia y Ecuador en el tránsito del neoliberalismo al postneoliberalismo. Íconos. Revista de Ciencias Sociales, n. 44, p. 11-17, 2012.

CUNHA FILHO, C. M. 2008, o ano da virada de Evo Morales? Observador On-line, v. 3, n. 12, p. 2-17, 2008.

CUNHA FILHO, C. M. El "proceso de cambio" en Bolivia: un balance de ocho años. Tinkazos, v. 17, n. 35, p. 137-153, 2014.

CUNHA FILHO, C. M. O novo mapa político boliviano: uma interpretação a partir dos últimos resultados eleitorais. Observador On-line, v. 5, n. 6, p. 1-16, 2010.

DO ALTO, H.; STEFANONI, P. El MAS: las ambivalencias de la democracia corporativa. In: GARCÍA ORELLANA, L. A.; GARCÍA YAPUR, F. (Org.). Mutaciones del campo político en Bolivia. La Paz: PNUD-Bolivia, 2010. p. 303-363.

DOMINGO, P. Party politics, intermediation and representation. In: CRABTREE, J.; WHITEHEAD, L. (Org.). Towards democratic viability: the Bolivian experience. Basingstoke: Palgrave Macmillan, 2001. p. 141-159.

ERREJÓN, I.; CANELAS, M. Las Autonomías en Bolivia y su Horizonte: un análisis político. In: Ministerio De Autonomías. (Org.). Ensayos sobre la autonomía en Bolivia. La Paz: Ministerio de Autonomías, 2012. p. 21-32. (Serie Autonomías para la Gente).

FARTHING, L.; KOHL, B. Social control: Bolivia's new approach to coca reduction. Latin American Perspectives, v. 37, n. 4, p. 197-213, 2010. http:// dx.doi.org/10.1177/0094582X10372516.

FONTANA, L. B. On the perils and potentialities of revolution: conflict and collective action in contemporary Bolivia. Latin American Perspectives, v. 40, n. 3, p. 26-42, 2013. http://dx.doi.org/10.1177/0094582X13476003.

GARCÉS, V. F. Los Indigenas y su Estado (pluri)nacional? Una mirada al proceso constituyente boliviano. Cochabamba: CLACSO, 2013.

GARCÉS, V. F. Reflexiones constituyentes (notas de camino). Cochabamba: UMSS, 2012.

GUIMARÃES, C.; DOMINGUES, J. M.; MANEIRO, M. Bolívia - a história sem fim. In: DOMINGUES, J. M. et al. A Bolivia no espelho do futuro. Belo Horizonte: EDUFMG, 2009. p. 13-26.

GUTIÉRREZ AGUILAR, R. A. Los ritmos del Pachakuti: movilización y levantamiento indígena-popular en Bolivia. Buenos Aires: Tinta Limón, 2008. (Pensar en Movimiento, 6).

HARNECKER, M.; FUENTES, F. MAS-IPSP de Bolivia: instrumento político que surge de los movimientos sociales. Caracas: Centro Internacional Miranda, 2008. Disponible en: $<$ http://www.rebelion.org/docs/67155. pdf $>$. Acceso en: 27 apr. 2009. 
HOFMEISTER, W. Bolivia: la construcción de la democracia y la evolución del proceso político. In: HOFMEISTER, W. (Org.). Reformas politicas en América Latina. Rio de Janeiro: Fundação Konrad Adenauer, 2004. p. 65-101.

HYLTON, F.; THOMSON, S. Revolutionary horizons: past and present in Bolivian politics. London: Verso, 2007.

KOHL, B. H.; FARTHING, L. C. Impasse in Bolivia: neoliberal hegemony and popular resistance. London: Zed Books, 2006.

LINERA, Á. G. Las tensiones creativas de la revolución: la quinta fase del proceso de cambio. La Paz: Vicepresidencia del Estado, 2011.

MARTÍ I PUIG, S.; BASTIDAS, C. ¿Ha cambiado la protesta? La coyuntura actual de movilizaciones en Bolivia y Ecuador. Íconos. Revista de Ciencias Sociales, n. 44, p. 19-33, 2012.

MAYORGA, F. Dilemas: ensayos sobre democracia intercultural y estado plurinacional. La Paz: CESU, 2011.

MAYORGA, R. A. Bolivia's silent revolution. Journal of Democracy, v. 8, n. 1, p. 142-156, 1997. http:// dx.doi.org/10.1353/jod.1997.0006.

MESA GISBERT, C. D. Presidentes de Bolivia: entre urnas y fusiles. 4. ed. La Paz: Gisbert, 2006.

MOKRANI, D.; URIONA CRESPO, P. Construcción hegemónica o monopolización de la política: el Movimiento al Socialismo y las posibilidades del proceso de cambio. Observatorio Social de América Latina, v. XII, n. 29, p. 111-127, 2011.

MOLINA, F. ¿Por qué Evo Morales sigue siendo popular? Las fortalezas del MAS en la construcción de un nuevo orden. Nueva Sociedad, n. 245, p. 4-14, 2013.

MOLINA, F. El MAS en el centro de la política boliviana. In: GARCÍA ORELLANA, L. A.; GARCÍA YAPUR, F. (Org.). Mutaciones del campo político en Bolivia. La Paz: PNUD-Bolivia, 2010. p. 241-301.

MOLINA, F. Las implicaciones del tercer mandato de Evo Morales. La Paz: Infolatam, 2014. Disponible en: <http://www.infolatam.com/2014/10/13/lasimplicaciones-del-tercer-mandato-de-evo-morales/>. Acceso en: 13 oct. 2014.
OBSERVATÓRIO POLÍTICO SUL-AMERICANO. Banco de eventos OPSA. [S.1.]: Observatório Político SulAmericano, 2014. Disponible en: $<$ http://observatorio. iesp.uerj.br/banco.php>. Acceso en: 3 sep. 2014.

ORTIZ, J. Y. Bolivia: Del "proceso de cambio" a los cambios en el proceso. Bolivian Studies Journal/ Revista de Estudios Bolivianos, v. 18, p. 70-114, 2011.

QUIROGA, M. V. Somos nosotros, somos gobierno. Experiencia de movimientos sociales en Bolivia. Bolivian Studies Journal/Revista de Estudios Bolivianos, v. 17, p. 264-276, 2010.

ROSSELL, P. 2011: ¿el parteaguas del evismo? Bolivia después del conflicto del Tipnis. Nueva Sociedad, n. 237, p. 4-16, 2012.

SCHAVELZON, S. El nacimiento del Estado Plurinacional de Bolivia: etnografia de una Asamblea Constituyente. La Paz: CLACSO, 2012.

SCHILLING-VACAFLOR, A. Indigenous identities and politico-juridical demands of CSUTCB and CONAMAQ in the constitutional change process of Bolivia. T'inkazos, v. 4, Selected Edition, 2008.

SILVA, F. P. Equilibrios Precários: a trajetória do Movimento Ao Socialismo e seus dilemas. In: DOMINGUES, J. M. et al. (Org.). A Bolivia no espelho do futuro. Belo Horizonte: EDUFMG, 2009. p. 51-74.

TAPIA, L. El estado de derecho como tiranía. La Paz: CIDES-UMSA, 2011a.

TAPIA, L. Política salvaje. Buenos Aires: Waldhuter Editores, 2011b.

VARGAS, H.; KRUSE, T. Las victorias de Abril: una historia que aún no concluye. Observatorio Social de América Latina, n. 2, p. 7-14, 2000.

ZUAZO. M. ¿Cómo nació el MAS? La ruralización de la politica en Bolivia - entrevistas a 85 parlamentarios del partido. 2. ${ }^{a}$ ed. La Paz: Friedrich Ebert Stiftung, 2009.

Recebido: 04 dec., 2014 Aceito: 02 mar., 2015 